\title{
OSCILLATION FOR ADVANCED DIFFERENTIAL EQUATIONS WITH OSCILLATING COEFFICIENTS
}

\author{
XIANYI LI, DEMING ZHU, and HANQING WANG
}

Received 3 September 2002

Some sufficient conditions are established for the oscillation of all solutions of the advanced differential equation $x^{\prime}(t)-p(t) x(t+\tau)=0, t \geq t_{0}$, where the coefficient $p(t) \in C\left(\left[t_{0}, \infty\right), R\right)$ is oscillatory, and $\tau$ is a positive constant.

2000 Mathematics Subject Classification: 34C10, 33C15, 34K11.

1. Introduction. Consider the following advanced differential equation:

$$
x^{\prime}(t)-p(t) x(t+\tau)=0, \quad t \geq t_{0}
$$

where the coefficient $p(t) \in C\left(\left[t_{0}, \infty\right), R\right)$ is an oscillatory function, that is, $p(t)$ takes both positive and negative values, and $\tau$ is a positive constant.

The problem of establishing sufficient conditions for the oscillation of all solutions of (1.1) has been the subject of many investigations. For example, see $[1,2,3,4,5,6]$ and the references cited therein. With respect to the oscillation of difference equation with oscillating coefficients, readers can refer to $[7,8$, $9,10]$.

By a solution of (1.1), we mean a continuously differentiable function defined on $\left[t_{0}, \infty\right)$ such that (1.1) is satisfied for $t \geq t_{0}$. As is customary, a solution of (1.1) is called oscillatory if it has arbitrarily large zeros. Otherwise it is called nonoscillatory. Equation (1.1) is called oscillatory if all its solutions are oscillatory.

For (1.1), Ladas and Stavroulakis [4] proved that all solutions of (1.1) oscillate if

$$
p(t) \geq 0, \quad \liminf _{t \rightarrow \infty} \int_{t}^{t+\tau} p(s) d s>\frac{1}{e} .
$$

For the result, see also Kusano [3] and Koplatadze and Chanturiya [1]. Recently, Li and Zhu [6] improved the above result to the following form. 
THEOREM 1.1. Suppose that there exist a $t_{1}>t_{0}+\tau$ and a positive integer $k$ such that

$$
\begin{gathered}
p_{k}(t) \geq \frac{1}{e^{k}}, \quad q_{k}(t) \geq \frac{1}{e^{k}}, \quad t \geq t_{1}+k \tau, \\
\int_{t_{1}+k \tau}^{\infty} p(t)\left[\exp \left(e^{k-1} p_{k}(t)-\frac{1}{e}\right)-1\right] d t=\infty .
\end{gathered}
$$

Then every solution of (1.1) oscillates. Here, $p(t) \in C\left(\left[t_{0}, \infty\right),[0, \infty)\right)$ and the sequences $\left\{p_{n}(t)\right\}$ and $\left\{q_{n}(t)\right\}$ of functions are defined as follows:

$$
\begin{aligned}
& p_{1}(t)=\int_{t}^{t+\tau} p(s) d s, \\
& p_{n}(t)=\int_{t}^{t+\tau} p(s) p_{n-1}(s) d s, \quad n \geq 2, t \geq t_{0}, \\
& q_{1}(t)=\int_{t-\tau}^{t} p(s) d s, \quad t \geq t_{0}+\tau, \\
& q_{n}(t)=\int_{t-\tau}^{t} p(s) q_{n-1}(s) d s, \quad n \geq 2, t \geq t_{0}+n \tau .
\end{aligned}
$$

For the studies of the oscillation of (1.1), all the previous works, such as Ladas and Stavroulakis [4], Kusano [3], Li and Zhu [6], and Koplatadze and Chanturiya [1], are under the assumption that the coefficient $p(t)$ has constant sign, that is, $p(t) \in C\left(\left[t_{0}, \infty\right), R_{+}\right)$. These investigations, in general, make use of the observation that if $x(t)$ is an eventually positive solution of (1.1), then $x^{\prime}(t)=p(t) x(t+\tau) \geq 0$ for all large $t$ so that $x(t)$ is eventually nondecreasing. However, when the coefficient $p(t)$ is oscillatory, that is, $p(t)$ takes both positive and negative values, the monotonicity does not hold any longer. All known results in the literature cannot be applied to the case where the coefficient $p(t)$ is oscillatory. Then, a natural question arises on how to investigate the oscillation of (1.1) when the coefficient $p(t)$ is oscillatory.

This problem, to the best of our knowledge, does not have any results up to now. The aim of this paper is to solve the problem. Our work is the continuity of that in [6], and the result obtained is of significance because a large number of oscillation criteria for higher-order as well as nonlinear differential equations can be reduced to oscillation criteria for equations of form (1.1).

2. Main result. As a starting point, we introduce a lemma that is required for the proof of our main results.

LEMMA 2.1. Suppose that $r \geq 0$ and that $\varphi(\cdot)$ is a nonnegative function in $R$ with $\varphi(0)=0$. Then $\varphi(r) r e^{x} \geq \varphi(r) x+\varphi(r) \ln (e r+1-\operatorname{sign}(r))$ for any 
$x \in R$, where the function $\operatorname{sign}(\cdot)$ is the usual sign function, that is,

$$
\operatorname{sign}(r)= \begin{cases}-1 & r<0 \\ 0 & r=0 \\ 1 & r>0\end{cases}
$$

The proof of Lemma 2.1 is trivial and is omitted here.

REMARK 2.2. In Lemma 2.1, the condition " $\varphi(0)=0$ " is only required to ensure the inequality holding for $r=0$. When $r>0$, this condition is unnecessary.

The main result of this paper is the following.

THEOREM 2.3. Let $\left\{a_{n}\right\}_{n=1}^{\infty}$ and $\left\{b_{n}\right\}_{n=1}^{\infty}$ be two sequences in $\left[t_{0}, \infty\right)$ satisfying

$$
a_{n}+2 \tau \leq b_{n} \leq a_{n+1}-2 \tau
$$

Assume that

$$
p(t) \geq 0, \quad \text { for } t \in \cup_{n=1}^{\infty}\left[a_{n}, b_{n}\right] .
$$

Define function $P(t)$ as follows:

$$
P(t)= \begin{cases}p(t), & t \in \cup_{n=1}^{\infty}\left[a_{n}, b_{n}-\tau\right] \\ 0, & \text { otherwise. }\end{cases}
$$

If

$$
\int_{t_{0}}^{\infty} P(t) \ln \left[e \int_{t-\tau}^{t} P(s) d s+1-\operatorname{sign}\left(\int_{t-\tau}^{t} P(s) d s\right)\right] d t=\infty,
$$

then every solution of (1.1) is oscillatory.

Proof. Assume, for the sake of contradiction, that there exists an eventually positive solution $x(t)$ of (1.1). Without loss of generality, we suppose that $x(t)>0$ for $t \geq a_{2}$. With respect to function $P(t)$, we have the following assertions:

(i) $\int_{t-\tau}^{t} P(s) d s<1, t \geq a_{2}$;

(ii) $\lim _{t \rightarrow \infty} \sup \int_{t-\tau}^{t} P(s) d s>0$.

We now first prove (i). Indeed, according to the definition of $P(t)$, we derive

$$
0 \leq P(t) \leq p(t), \quad t \in \cup_{n=2}^{\infty}\left[a_{n}, b_{n}\right] .
$$

Then, by (1.1), one can see

$$
x^{\prime}(t)-P(t) x(t+\tau) \geq 0, \quad t \in \cup_{n=2}^{\infty}\left[a_{n}, b_{n}\right],
$$

and so $x(t)$ is nondecreasing for $t \in \cup_{n=2}^{\infty}\left[a_{n}, b_{n}\right]$. 
If $t \in \cup_{n=2}^{\infty}\left[a_{n}+\tau, b_{n}-\tau\right]$, then $[t-\tau, t] \subset \cup_{n=2}^{\infty}\left[a_{n}, b_{n}-\tau\right]$. Integrating (2.7) from $t-\tau$ to $t$ and noticing the nondecreasing nature of $x(t)$ give

$$
\begin{aligned}
x(t) & \geq x(t-\tau)+\int_{t-\tau}^{t} P(s) x(s+\tau) d s \\
& >\int_{t-\tau}^{t} P(s) x(s+\tau) d s \\
& \geq x(t) \int_{t-\tau}^{t} P(s) d s,
\end{aligned}
$$

which implies

$$
\int_{t-\tau}^{t} P(s) d s<1, \quad t \in \cup_{n=2}^{\infty}\left[a_{n}+\tau, b_{n}-\tau\right]
$$

If $t \notin \cup_{n=2}^{\infty}\left[a_{n}+\tau, b_{n}-\tau\right]$, then one can consider the following three cases.

CASE 1. If $t \in \cup_{n=2}^{\infty}\left(b_{n}-\tau, b_{n}\right]$, then, by definition (2.4) of $P(t)$,

$$
\begin{aligned}
\int_{t-\tau}^{t} P(s) d s & \leq \int_{b_{n}-2 \tau}^{t} P(s) d s \\
& =\int_{b_{n}-2 \tau}^{b_{n}-\tau} P(s) d s+\int_{b_{n}-\tau}^{t} P(s) d s \\
& =\int_{b_{n}-2 \tau}^{b_{n}-\tau} P(s) d s<1 .
\end{aligned}
$$

Note that (2.9) is used in getting the last inequality above.

CASE 2. If $t \in \cup_{n=2}^{\infty}\left[a_{n}, a_{n}+\tau\right)$, then

$$
\int_{t-\tau}^{t} P(s) d s=\int_{t-\tau}^{a_{n}} P(s) d s+\int_{a_{n}}^{t} P(s) d s=\int_{a_{n}}^{t} P(s) d s \leq \int_{a_{n}}^{a_{n}+\tau} P(s) d s<1 .
$$

CASE 3. If $t \in \cup_{n=2}^{\infty}\left(b_{n}, a_{n+1}\right]$, then

$$
\int_{t-\tau}^{t} P(s) d s=0<1 .
$$

Thus, we complete the proof of assertion (i).

Next, we will show assertion (ii). If we have $\lim _{t \rightarrow \infty} \int_{t-\tau}^{t} P(s) d s=0$, so there exists a $T \geq t_{0}$ such that

$$
\int_{t-\tau}^{t} P(t) d t<\frac{1}{e} \quad \text { for } t \geq T
$$

It follows from (2.5) that

$$
\int_{a_{2}}^{\infty} P(t) \ln \left[e \int_{t-\tau}^{t} P(s) d s+1-\operatorname{sign}\left(\int_{t-\tau}^{t} P(s) d s\right)\right] d t=\infty .
$$


Therefore,

$$
\begin{aligned}
\infty & =\int_{a_{2}}^{\infty} P(t) \ln \left[e \int_{t-\tau}^{t} P(s) d s+1-\operatorname{sign}\left(\int_{t-\tau}^{t} P(s) d s\right)\right] d t \\
& =\sum_{n=2}^{\infty} \int_{a_{n}}^{a_{n+1}} P(t) \ln \left[e \int_{t-\tau}^{t} P(s) d s+1-\operatorname{sign}\left(\int_{t-\tau}^{t} P(s) d s\right)\right] d t \\
& =\sum_{n=2}^{\infty}\left(\int_{a_{n}}^{b_{n}-\tau}+\int_{b_{n-\tau}}^{a_{n+1}}\right) P(t) \ln \left[e \int_{t-\tau}^{t} P(s) d s+1-\operatorname{sign}\left(\int_{t-\tau}^{t} P(s) d s\right)\right] d t \\
& =\sum_{n=2}^{\infty} \int_{a_{n}}^{b_{n}-\tau} P(t) \ln \left[e \int_{t-\tau}^{t} P(s) d s+1-\operatorname{sign}\left(\int_{t-\tau}^{t} P(s) d s\right)\right] d t .
\end{aligned}
$$

And so there exists a positive integer sequence $\left\{n_{i}\right\}_{i=1}^{\infty}$ such that

$$
\int_{a_{n_{i}}}^{b_{n_{i}}-\tau} P(t) \ln \left[e \int_{t-\tau}^{t} P(s) d s+1-\operatorname{sign}\left(\int_{t-\tau}^{t} P(s) d s\right)\right] d t>0, \quad i=1,2, \ldots
$$

Since $P(t)$ is nonnegative and continuous in $t \in\left[a_{n_{i}}, b_{n_{i}}-\tau\right], i \geq 1$, there exists an interval $\left[k_{i}, l_{i}\right] \subset\left[a_{n_{i}}, b_{n_{i}}-\tau\right], i \geq 1$, such that

$P(t) \ln \left[e \int_{t-\tau}^{t} P(s) d s+1-\operatorname{sign}\left(\int_{t-\tau}^{t} P(s) d s\right)\right] d t>0, \quad t \in\left[k_{i}, l_{i}\right], i=1,2, \ldots$

From this, we know

$$
\int_{t-\tau}^{t} P(t) d t>\frac{1}{e}, \quad t \in\left[k_{i}, l_{i}\right], i=1,2, \ldots
$$

which is contrary to (2.13). So, assertion (ii) is true. Accordingly, there exist a constant $d>0$ and a sequence $\left\{t_{n}\right\}$ of points such that $t_{n} \rightarrow \infty$ as $n \rightarrow \infty$, $t_{n} \in\left[a_{n}, b_{n}-\tau\right]$, and

$$
\int_{t_{n-\tau}}^{t_{n}} P(t) d t \geq d, \quad n=1,2, \ldots
$$

We now set

$$
\lambda(t)=\frac{x^{\prime}(t)}{x(t)}, \quad t \geq a_{2}
$$

Then, by (2.7),

$$
\lambda(t) \geq 0, \quad t \in \cup_{n=2}^{\infty}\left[a_{n}, b_{n}\right]
$$


Integrating (2.20) from $t \geq a_{2}$ to $t+\tau$ and noting (1.1), one can obtain

$$
\begin{gathered}
\lambda(t)=p(t) \exp \left(\int_{t}^{t+\tau} \lambda(s) d s\right), \quad t \geq a_{2}, \\
\lambda(t) \int_{t-\tau}^{t} P(s) d s=p(t) \int_{t-\tau}^{t} P(s) d s \exp \left(\int_{t}^{t+\tau} \lambda(s) d s\right), \quad t \geq a_{2} .
\end{gathered}
$$

Notice that $P(t)$ is nonnegative and continuous restricted to $\cup_{n=2}^{\infty}\left[a_{n}, b_{n}-\tau\right]$. We can show that $\int_{t-\tau}^{t} P(s) d s=0$ for any $t \in \cup_{n=2}^{\infty}\left[a_{n}, b_{n}-\tau\right]$ is equivalent to $P(t)=0$ for any $t \in \cup_{n=2}^{\infty}\left[a_{n}, b_{n}-\tau\right]$. Now, we simply verify this as follows.

If $\int_{t-\tau}^{t} P(s) d s=0$ for any $t \in \cup_{n=2}^{\infty}\left[a_{n}, b_{n}-\tau\right]$, then there must be $P(t)=0$ for any $t \in \cup_{n=2}^{\infty}\left[a_{n}, b_{n}-\tau\right]$. We only prove $P(t)=0$ at the point $a_{n}$ and the proofs for the other points are similar and are omitted. Otherwise, $P\left(a_{n}\right)>$ 0 . Since $P(t)$ is right-continuous at the point $a_{n}$, there exists a $\delta>0$ such that $P(t)>0$ for $t \in\left[a_{n}, a_{n}+\delta\right)$. Hence, for any given $\epsilon \in(0, \min \{\tau, \delta\})$, $\int_{a_{n}+\epsilon-\tau}^{a_{n}+\epsilon} P(s) d s=\int_{a_{n}}^{a_{n}+\epsilon} P(s) d s>0$, which contradicts the known assumption.

Conversely, if $P(t)=0$ for any $t \in \cup_{n=2}^{\infty}\left[a_{n}, b_{n}-\tau\right]$, then, according to definition (2.4) of $P(t)$, one can see that $P(t) \equiv 0$ for $t \geq a_{2}$, which means that $\int_{t-\tau}^{t} P(s) d s=0$ for any $t \in \cup_{n=2}^{\infty}\left[a_{n}, b_{n}-\tau\right]$, and so the conclusion above holds.

Therefore, by using Lemma 2.1 with $r=\int_{t-\tau}^{t} P(s) d s, \varphi(r)=P(t)$, and $x=$ $\int_{t}^{t+\tau} \lambda(s) d s$, we derive

$$
\begin{aligned}
& \lambda(t) \int_{t-\tau}^{t} P(s) d s \\
& \quad \geq P(t) \int_{t-\tau}^{t} P(s) d s \exp \left(\int_{t}^{t+\tau} \lambda(s) d s\right) \\
& \quad \geq P(t) \int_{t}^{t+\tau} \lambda(s) d s+P(t) \ln \left[e \int_{t-\tau}^{t} P(s) d s+1-\operatorname{sign}\left(\int_{t-\tau}^{t} P(s) d s\right)\right],
\end{aligned}
$$

that is, for $t \in \cup_{n=2}^{\infty}\left[a_{n}, b_{n}-\tau\right]$,

$$
\begin{aligned}
& \lambda(t) \int_{t-\tau}^{t} P(s) d s \\
& \quad \geq P(t) \int_{t}^{t+\tau} \lambda(s) d s+P(t) \ln \left[e \int_{t-\tau}^{t} P(s) d s+1-\operatorname{sign}\left(\int_{t-\tau}^{t} P(s) d s\right)\right] .
\end{aligned}
$$

Notice that $P(t)=0$ for $t \in \cup_{n=2}^{\infty}\left(b_{n}-\tau, a_{n+1}\right)$. Accordingly, we have, for $t \in$ $\cup_{n=2}^{\infty}\left(b_{n}-\tau, b_{n}\right], \int_{t-\tau}^{t} P(s) d s=\int_{t-\tau}^{b_{n}-\tau} P(s) d s>0$. This, together with (2.23) and Lemma 2.1, implies the validity of (2.25). For $t \in \cup_{n=2}^{\infty}\left(b_{n}, a_{n+1}\right), \int_{t-\tau}^{t} P(s) d s=$ 0 , which also indicates that (2.25) holds. So, (2.25) is true for $t \geq a_{2}$. 
Integrating (2.25) from $a_{2}$ to $t_{n}$ for $n>2$ produces

$$
\begin{aligned}
& \int_{a_{2}}^{t_{n}} \lambda(t) \int_{t-\tau}^{t} P(s) d s d t-\int_{a_{2}}^{t_{n}} P(t) \int_{t}^{t+\tau} \lambda(s) d s d t \\
& \quad \geq \int_{a_{2}}^{t_{n}} P(t) \ln \left[e \int_{t-\tau}^{t} P(s) d s+1-\operatorname{sign}\left(\int_{t-\tau}^{t} P(s) d s\right)\right] d t .
\end{aligned}
$$

Put

$$
\begin{aligned}
& D_{1}=\left\{(s, t) \mid a_{2}+\tau \leq s \leq t_{n}, s-\tau \leq t \leq s\right\}, \\
& D_{2}=\left\{(s, t) \mid t_{n} \leq s \leq t_{n}+\tau, s-\tau \leq t \leq t_{n}\right\}, \\
& D_{3}=\left\{(s, t) \mid a_{2} \leq t \leq a_{2}+\tau, t \leq s \leq a_{2}+\tau\right\} .
\end{aligned}
$$

It is clear that $P(t) \lambda(s) \geq 0$ for $(t, s) \in D_{2} \cup D_{3}$. Thus,

$$
\begin{aligned}
\int_{a_{2}}^{t_{n}} P(t) \int_{t}^{t+\tau} \lambda(s) d s d t & =\sum_{i=1}^{3} \iint_{D_{i}} P(t) \lambda(s) d s d t \\
& \geq \iint_{D_{1}} P(t) \lambda(s) d s d t=\int_{a_{2}+\tau}^{t_{n}} \int_{s-\tau}^{s} P(t) \lambda(s) d s d t \\
& =\int_{a_{2}+\tau}^{t_{n}} \lambda(s) \int_{s-\tau}^{s} P(t) d t d s=\int_{a_{2}+\tau}^{t_{n}} \lambda(t) \int_{t-\tau}^{t} P(s) d s d t
\end{aligned}
$$

This, together with (2.26), leads to

$$
\begin{array}{rl}
\int_{a_{2}}^{t_{n}} & P(t) \ln \left[e \int_{t-\tau}^{t} P(s) d s+1-\operatorname{sign}\left(\int_{t-\tau}^{t} P(s) d s\right)\right] d t \\
& \leq \int_{a_{2}}^{t_{n}} \lambda(t) \int_{t-\tau}^{t} P(s) d s d t-\int_{a_{2}+\tau}^{t_{n}} \lambda(t) \int_{t-\tau}^{t} P(s) d s d t \\
& =\int_{a_{2}}^{a_{2}+\tau} \lambda(t) \int_{t-\tau}^{t} P(s) d s d t<\infty,
\end{array}
$$

whereas, according to (2.5), we have

$$
\lim _{n \rightarrow \infty} \int_{a_{2}}^{t_{n}} P(t) \ln \left[e \int_{t-\tau}^{t} P(s) d s+1-\operatorname{sign}\left(\int_{t-\tau}^{t} P(s) d s\right)\right] d t=\infty,
$$

which contradicts (2.29) and completes the proof. 
3. Example. As an application of Theorem 2.3, we consider the oscillation of the following equation:

$$
x^{\prime}(t)-p(t) x(t+1)=0, \quad t \geq 0,
$$

where $\tau=1$ and the function $p(t)$ is 6-periodic one with

$$
p(t)= \begin{cases}-t, & 0 \leq t \leq 1 \\ t-2, & 1<t \leq 4 \\ 6-t, & 4<t \leq 6\end{cases}
$$

Obviously, $\lim _{t \rightarrow \infty} \inf \int_{t}^{t+\tau} p(t) d s=-1 / 2<0$. Therefore, the results in [1, 2, 3, $4,5,6]$ cannot be applied to (3.1). But, if we denote $a_{n}=2+6(n-1), b_{n}=6 n$, $n \geq 1$, then, clearly, $a_{n}, b_{n} \in[0, \infty)$,

$$
a_{n}+2 \tau \leq b_{n} \leq a_{n+1}-2 \tau, \quad n=1,2, \ldots,
$$

and $p(t) \geq 0$ for $t \in \cup_{n=1}^{\infty}\left[a_{n}, b_{n}\right]$. Furthermore, if we set

$$
P(t)= \begin{cases}p(t), & t \in \cup_{n=1}^{\infty}\left[a_{n}, b_{n}-\tau\right], \\ 0, & \text { otherwise }\end{cases}
$$

then we have

$$
\begin{aligned}
\int_{a_{n}}^{b_{n}-\tau} P(t) \ln \left[e \int_{t-\tau}^{t} P(s) d s+1-\operatorname{sign}\left(\int_{t-\tau}^{t} P(s) d s\right)\right] d t \\
=\int_{2}^{5} P(t) \ln \left[e \int_{t-\tau}^{t} P(s) d s+1-\operatorname{sign}\left(\int_{t-\tau}^{t} P(s) d s\right)\right] d t \\
=\int_{2}^{4} P(t) \ln \left[e \int_{t-\tau}^{t} P(s) d s+1-\operatorname{sign}\left(\int_{t-\tau}^{t} P(s) d s\right)\right] d t \\
+\int_{4}^{5} P(t) \ln \left[e \int_{t-\tau}^{t} P(s) d s+1-\operatorname{sign}\left(\int_{t-\tau}^{t} P(s) d s\right)\right] d t \\
=\int_{2}^{4}(t-2) \ln \left[e\left(\int_{t-1}^{2} P(s) d s+\int_{2}^{t} P(s) d s\right)+1-\operatorname{sign}\left(\int_{t-\tau}^{t} P(s) d s\right)\right] d t \\
+\int_{4}^{5}(6-t) \ln \left[e\left(\int_{t-1}^{2} P(s) d s+\int_{2}^{4} P(s) d s+\int_{4}^{t} P(s) d s\right)\right.
\end{aligned}
$$




$$
\begin{aligned}
= & \int_{2}^{4}(t-2) \ln \left(e \int_{2}^{t}(s-2) d s\right) d t+\int_{4}^{5}(6-t) \ln \left(e\left(2+\int_{4}^{t}(6-s) d s\right)\right) d t \\
= & {\left.\left[\int_{2}^{t}(s-2) d s \ln \left(e \int_{2}^{t}(s-2)\right) d s-\int_{2}^{t}(s-2) d s\right]\right|_{2} ^{4} } \\
& +\left.\left[\left(2+\int_{4}^{t}(6-s) d s\right) \ln \left(e\left(2+\int_{4}^{t}(6-s) d s\right)\right)-\left(2+\int_{4}^{t}(6-s) d s\right)\right]\right|_{4} ^{5} \\
= & 2 \ln 2+\left(\frac{7}{2} \ln \frac{7}{2}-2 \ln 2\right)=\frac{7}{2} \ln \frac{7}{2}>0
\end{aligned}
$$

which means that

$$
\int_{a_{2}}^{\infty} P(t) \ln \left[e \int_{t-\tau}^{t} P(s) d s+1-\operatorname{sign}\left(\int_{t-\tau}^{t} P(s) d s\right)\right] d t=\infty
$$

By Theorem 2.3, every solution of (3.1) is oscillatory.

ACKNOWLEDGMENT. This work was supported by NNSFC, Grant 10071022, Mathematical Tianyuan Foundation of China, Grant TY10026002-01-05-03, and Shanghai Priority Academic Discipline.

\section{REFERENCES}

[1] R. G. Koplatadze and T. A. Chanturiya, Oscillating and monotone solutions of first-order differential equations with deviating argument, Differentsial'nye Uravneniya 18 (1982), no. 8, 1463-1465.

[2] M. R. Kulenović and M. K. Grammatikopoulos, Some comparison and oscillation results for first-order differential equations and inequalities with a deviating argument, J. Math. Anal. Appl. 131 (1988), no. 1, 67-84.

[3] T. Kusano, On even-order functional-differential equations with advanced and retarded arguments, J. Differential Equations 45 (1982), no. 1, 75-84.

[4] G. Ladas and I. P. Stavroulakis, Oscillations caused by several retarded and advanced arguments, J. Differential Equations 44 (1982), no. 1, 134-152.

[5] G. S. Ladde, V. Lakshmikantham, and B. G. Zhang, Oscillation Theory of Differential Equations with Deviating Arguments, Monographs and Textbooks in Pure and Applied Mathematics, vol. 110, Marcel Dekker, New York, 1987.

[6] X. Li and D. Zhu, Oscillation and nonoscillation of advanced differential equations with variable coefficients, J. Math. Anal. Appl. 269 (2002), no. 2, 462-488.

[7] X.-H. Tang and S. S. Cheng, An oscillation criterion for linear difference equations with oscillating coefficients, J. Comput. Appl. Math. 132 (2001), no. 2, 319329.

[8] W. P. Yan and J. R. Yan, Comparison and oscillation results for delay difference equations with oscillating coefficients, Int. J. Math. Math. Sci. 19 (1996), no. 1, 171-176. 
[9] J. S. Yu, B. G. Zhang, and X. Z. Qian, Oscillations of delay difference equations with oscillating coefficients, J. Math. Anal. Appl. 177 (1993), no. 2, 432-444.

[10] G. Zhang and S. S. Cheng, Elementary oscillation criteria for a three term recurrence relation with oscillatory coefficient sequence, Tamkang J. Math. 29 (1998), no. 3, 227-232.

Xianyi Li: School of Mathematics and Physics, Nanhua University, Hengyang 421001, China

Current address: Department of Mathematics, East China Normal University, Shanghai 200062, China

E-mail address: xianyi1i6590@sina.com.cn

Deming Zhu: Department of Mathematics, East China Normal University, Shanghai 200062, China

E-mail address: dmzhu@math. ecnu. edu.cn

Hanqing Wang: School of Architectural Engineering, Resources and Environment, Nanhua University, Hengyang 421001, China

E-mail address: hqwang888@sina.com.cn 


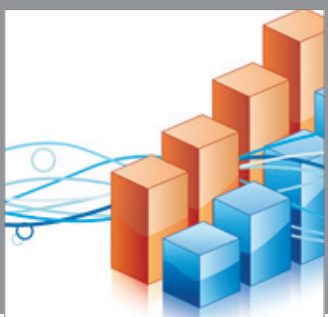

Advances in

Operations Research

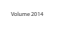

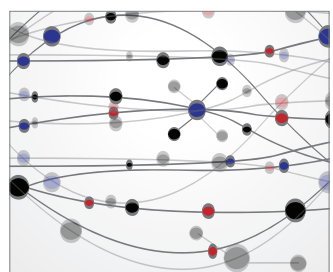

\section{The Scientific} World Journal
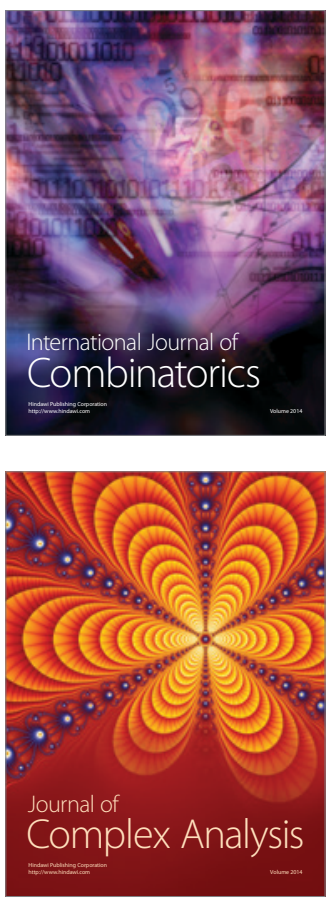

International Journal of

Mathematics and

Mathematical

Sciences
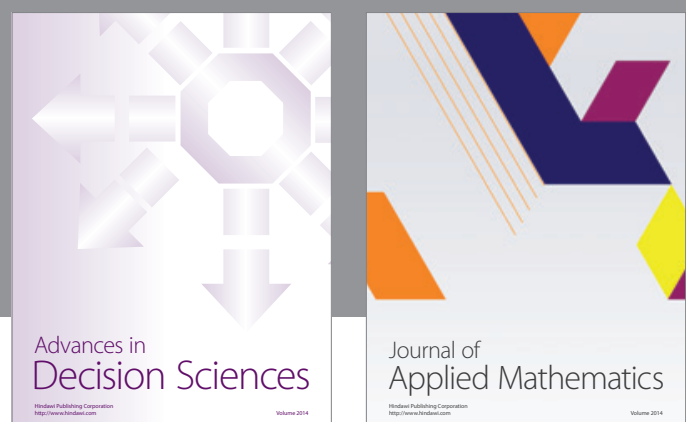

Journal of

Applied Mathematics
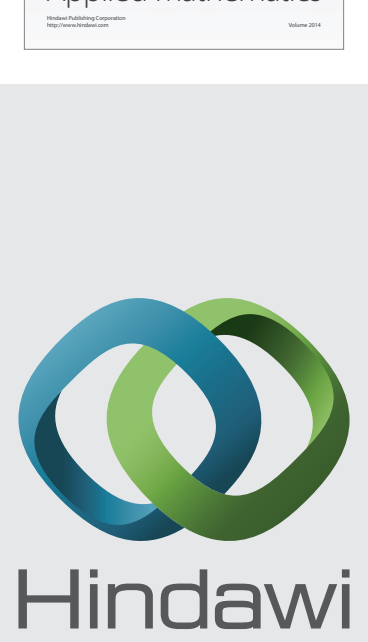

Submit your manuscripts at http://www.hindawi.com
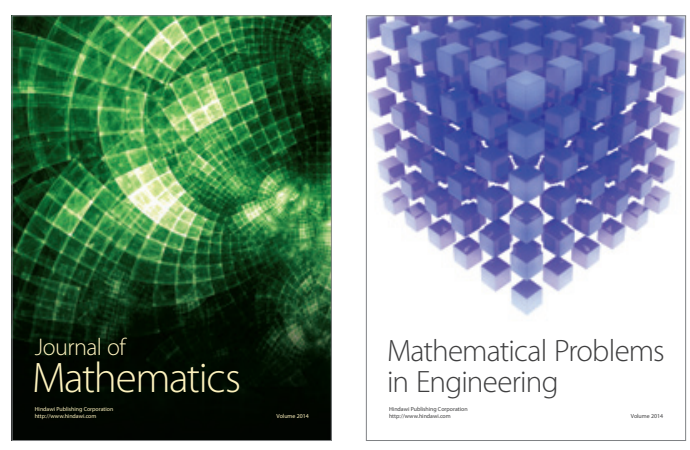

Mathematical Problems in Engineering
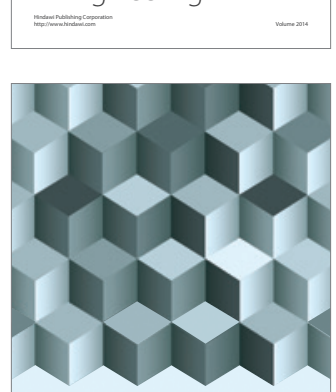

Journal of

Function Spaces
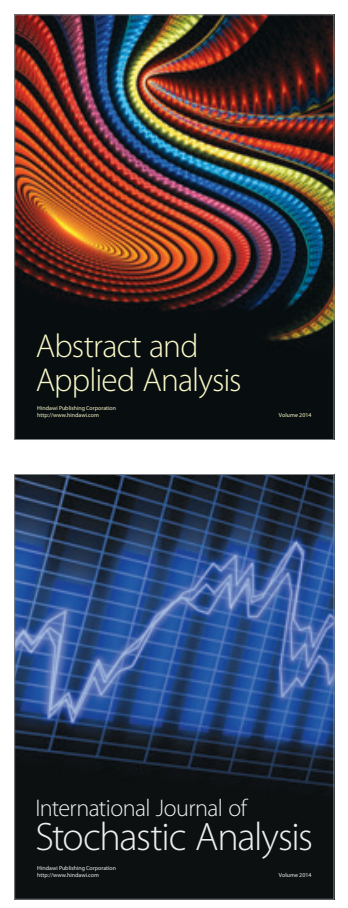

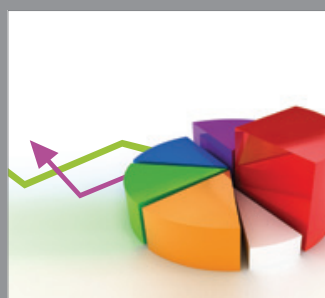

ournal of

Probability and Statistics

Promensencen
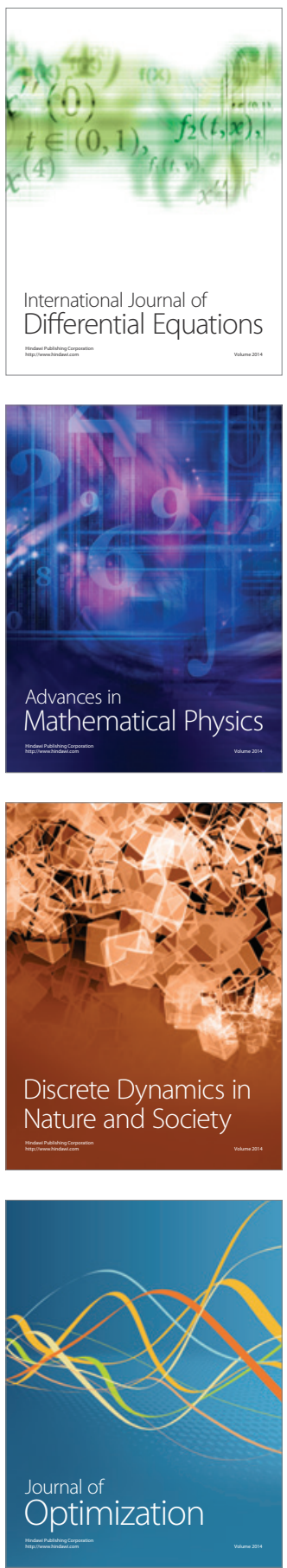